\title{
Dynamics of multiple interacting excitatory and inhibitory populations with delays
}

\author{
Christopher M. Kim, ${ }^{1, *}$ Ulrich Egert, ${ }^{1,2}$ and Arvind Kumar ${ }^{1,3, \dagger}$ \\ ${ }^{1}$ Bernstein Center Freiburg, Freiburg, Germany \\ ${ }^{2}$ Biomicrotechnology, IMTEK - Department of Microsystems Engineering, University of Freiburg, Freiburg, Germany \\ ${ }^{3}$ Department of Computational Science and Technology, \\ School for Electrical Engineering and Computer Science KTH Royal Institute of Technology, Stockholm, Sweden
}

(Dated: June 27, 2018)

\begin{abstract}
A network consisting of excitatory and inhibitory (EI) neurons is a canonical model for understanding cortical network activity. In this study, we extend the EI network model and investigate how its dynamical landscape can be enriched when it interacts with another excitatory (E) population with transmission delays. Through analysis and simulations of a rate model and a spiking network model, we study the transition from stationary to oscillatory states by analyzing the Hopf bifurcation structure in terms of two network parameters: 1) transmission delay between the EI subnetwork and the E population and 2) inhibitory couplings that induce oscillatory activity in the EI subnetwork. We find that the critical coupling strength can strongly modulate as a function of transmission delay, and consequently the stationary state is interwoven intricately with oscillatory states generating different frequency modes. This leads to the emergence of an isolated stationary state surrounded by multiple oscillatory states and cross-frequency coupling develops at the bifurcation points. We identify the possible network motifs that induce oscillations and examine how multiple oscillatory states come together to enrich the dynamical landscape.
\end{abstract}

\section{INTRODUCTION}

The brain is organized as a network of highly specialized subnetworks. Each of the subnetworks consists of a large number of excitatory and inhibitory neurons communicating via spikes. Randomly connected networks of excitatory and inhibitory neurons have been a popular and useful model to study the dynamical states and information processing in local networks of the brain. Previous work has demonstrated that balance of excitation and inhibition (EI-balance) is a crucial variable that determines two qualitatively different states of global network activity. When excitation and inhibition are balanced, cancellation of excitatory and inhibitory synaptic inputs to a neuron leads to asynchronous and nearly Poisson type spiking $[1,2]$. A mismatch between excitation and inhibition (in amplitude or timing) results in oscillatory states [3-5], in which the population firing rate oscillates while individual neurons spike irregularly. Both network states are considered to play important roles in cortical processing; the asynchronous activity of the balanced state provides a suitable substrate to perform complex computations [6,7], balanced amplification [8,9] and propagation of rate and time coded signals [10], and oscillatory rhythms play a crucial role in selective routing information across multiple brain areas [1114].

Besides the EI-balance, spike propagation time delays introduce various complex effects on the network activity dynamics. For instance, delays may destabilize the balanced state of spiking networks [3-5], enrich the bifurcation structure of spatially-extended neural field models by introducing

\footnotetext{
* Corresponding author 1: chrismkkim @gmail.com;

Current address: Laboratory of Biological Modeling, NIDDK/NIH, Bethesda, MD, USA

† Corresponding author 2: arvkumar@kth.se
}

novel dynamical states $[15,16]$ and gate the propagation of spiking activity [17]. Moreover, by suitably tuning the delays oscillations can be enhanced and suppressed [18-20].

Here we go beyond the standard two population (one excitatory and one inhibitory) model of local cortical networks and investigate how the addition of one more excitatory population alters the dynamical landscape of the standard excitatoryinhibitory (EI) spiking networks composed of balanced and oscillatory states. The third excitatory population is coupled to standard EI network with a longer delay as compared to the delays within in the EI network. Thus, this model allows us to understand how long and short delays interact to shape the critical coupling strength between excitatory-inhibitory or inhibitory-inhibitory neurons that generate network-wide oscillations [3-5].

Through analysis and simulations of the three-population network models, we show that the balanced state can be interwoven intricately with multiple oscillatory states when the EI network is strongly coupled to a third excitatory population with delays. Such dynamical landscapes naturally give rise to cross-frequency oscillations in parameter regime where multiple oscillatory states merge. Our study demonstrates the rich dynamic repertoire of interacting sub-networks in the presence of delays and paves the way to study a network of many sub-networks.

\section{NETWORK MODEL}

The network consisted of two excitatory $\left(E_{1}, E_{2}\right)$ and one inhibitory $\left(I_{3}\right)$ populations. The $E_{2} I_{3}$ subnetwork was the standard EI network [3] which was reciprocally connected to the $E_{1}$ population (Fig. 1). We did not include recurrent excitatory connections within $E_{1}$ and $E_{2}$ because we focused on oscillatory network activity generated by the excitatoryinhibitory and inhibitory-inhibitory couplings. We refer to the connectivity parameters between $E_{1}$ and $E_{2} I_{3}$ "lateral", and 


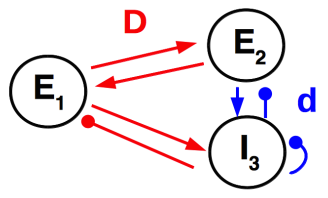

FIG. 1 . The connectivity structure of $E_{1} E_{2} I_{3}$ network.

those within the $E_{2} I_{3}$ subnetwork "local". The population $E_{1}$ was considered to be located at a farther distance; therefore, the transmission delay $D$ between $E_{1}$ and $E_{2} I_{3}$ was larger than the transmission delay $d$ within the $E_{2} I_{3}$ subnetwork. In this work we investigated the effect of long transmission delays and inhibitory connections from $I_{3}$ to $E_{1}, E_{2}$ and itself on network oscillations.

To study the network dynamics analytically, we considered a rate model that describes the firing rate dynamics of three populations and compared the results with numerical simulations of a comparable network with spiking neurons.

Firing rate model. The rate model was described as a set of delay differential equations,

$$
\begin{aligned}
& \tau_{1} \dot{r}_{1}(t)=-r_{1}(t)+\left[J_{11} s_{11}(t)+J_{12} s_{12}(t)-J_{13} s_{13}(t)+I_{1}\right]_{+} \\
& \tau_{2} \dot{r}_{2}(t)=-r_{2}(t)+\left[J_{21} s_{21}(t)+J_{22} s_{22}(t)-J_{23} s_{23}(t)+I_{2}\right]_{+} \\
& \tau_{3} \dot{r}_{3}(t)=-r_{3}(t)+\left[J_{31} s_{31}(t)+J_{32} s_{32}(t)-J_{33} s_{33}(t)+I_{3}\right]_{+},
\end{aligned}
$$

where $J_{a b}$ is the coupling strength of connection from population $b$ to population $a, I_{a}$ is an external input, and the activation function $[x]_{+}=x$ if $x>0$ and $=0$ otherwise. We let $J_{11}=J_{22}=0$ because there were no recurrent excitatory connections. The dynamics of synaptic current from $b$ to $a$ obeyed

$$
\tau_{d} \dot{s}_{a b}=-s_{a b}+r_{b}\left(t-D_{a b}\right) \quad a, b \in\{1,2,3\},
$$

where $\tau_{d}$ is a decay time constant and $D_{a b}$ is a transmission delay from $b$ to $a$.

The connections between $E_{1}$ and $E_{2} I_{3}$ had a transmission delay, $D$, and the connections within the $E_{2} I_{3}$ subnetwork had a transmission delay, $d ; D=D_{12}=D_{21}=D_{13}=D_{31}$ and $d=D_{23}=D_{32}=D_{33}$.

Network model with spiking neurons. For the spiking network model, we considered a network of randomly connected leaky integrate-and-fire (LIF) neurons where $E_{1}, E_{2}$ and $I_{3}$ population consists of $N_{1}=N / 2, N_{2}=N$ and $N_{3}=N / 4$ neurons $(N=10,000)$, respectively. The membrane potential of neuron $i$ in population $a$ obeyed

$$
\begin{aligned}
\tau_{m a} \dot{V}_{i}(t) & =V_{r}-V_{i}(t)+\mu_{a}+\sigma_{a} \xi_{i}(t) \\
& +\frac{J_{a 1}}{p N_{1}} \sum_{j \in E_{1}} s_{i j}+\frac{J_{a 2}}{p N_{2}} \sum_{j \in E_{2}} s_{i j}-\frac{J_{a 3}}{p N_{3}} \sum_{j \in I_{3}} s_{i j}
\end{aligned}
$$

and elicited an action potential when $V_{i}$ reached a threshold $V_{t h}$. Here, $\tau_{m a}$ is a membrane time constant, $V_{r}$ is a resting potential, $J_{a b}$ is total postsynaptic potential of synaptic connections from population $b$ to population $a, \mu_{a}$ is an external input, and $\sigma_{a} \xi_{i}$ is Gaussian white noise with mean zero and variance $\sigma_{a}^{2}$.

Every neuron received the same number $p N_{a}$ of recurrent synaptic inputs from randomly selected neurons in populations $a=1,2,3$. The strength of individual synapses from neuron $i$ in population $a$ to neuron $j$ in population $b$ was given by $J_{a b} /\left(p N_{b}\right)$

The synaptic current $s_{i j}$ decayed exponentially upon receiving a spike from a presynaptic neuron $j$ in population $b$.

$$
\tau_{d} \dot{s}_{i j}=-s_{i j}+\tau_{m a} \sum_{t_{i}^{k}<t} \delta\left(t-t_{i}^{k}-D_{a b}\right)
$$

where $t_{i}^{k}$ is the spike-time of presynaptic neurons, and $D_{a b}$ is the transmission delay from neurons in population $b$ to neurons in population $a$. As in the firing rate model, the connections between $E_{1}$ and $E_{2} I_{3}$ had a transmission delay, $D$, and the connections within the $E_{2} I_{3}$ subnetwork had a transmission delay, $d$.

Following the previous studies $[3,5,6]$, we estimated the steady-state firing rate using the Fokker-Planck approach, which meant solving a system of three nonlinear equations in a self-consistent manner.

$$
r_{a}=\Psi_{a}\left(X_{a}, Y_{a}\right), \quad a=1,2,3
$$

where

$$
\begin{aligned}
\Psi_{a}\left(X_{a}, Y_{a}\right) & =\left[\tau_{m a} \sqrt{\pi} \int_{\left(V_{r}-X_{a}\right) / Y_{a}}^{\left(V_{t h}-X_{a}\right) / Y_{a}} e^{t^{2}}(1+\operatorname{erf}(t)) d t\right]^{-1} \\
X_{a} & =\mu_{a}+J_{a 1} r_{1}+J_{a 2} r_{2}-J_{a 3} r_{3} \\
Y_{a} & =\sqrt{\sigma_{a}^{2}+J_{a 1}^{2} r_{1}+J_{a 2}^{2} r_{2}+J_{a 3}^{2} r_{3}} .
\end{aligned}
$$

Here, $r_{a}$ is the population-averaged firing rate, $X_{a}$ is the mean synaptic input, and $Y_{a}^{2}$ is the variance of total synaptic input to a neuron.

In the following network simulations, we adjusted the mean $\left(\mu_{a}\right)$ and variance $\left(\sigma_{a}^{2}\right)$ of external inputs to obtain the same steady-state firing rate $\left(r_{e}=5 \mathrm{~Hz}, r_{i}=10 \mathrm{~Hz}\right)$ for different network configurations. The external inputs $\left(I_{a}\right)$ to the rate model was adjusted similarly to maintain same $r_{a}$ across simulations. Firing rate equations (Eq. 1) were solved using Matlab's delay differential equation solver, dde23. The simulation of networks with spiking neurons was performed using the NEST simulation tool [21].

\section{DYNAMICS OF OSCILLATIONS IN THE THREE POPULATION MODEL}

\section{A. Linear stability of the steady-state}

To characterize how the lateral delay $D$ affects the emergence of oscillatory activity within the $E_{2} I_{3}$ subnetwork, we 
performed linear stability analysis of the steady-state of the rate model and the spiking network model. We added a small perturbation term to the steady-state firing rate such that $r_{a}(t)=r_{a 0}+\delta r_{a} \cdot e^{\lambda t}$. The rate perturbation induced a perturbation in the synaptic current:

$$
\delta s_{a b}(t)=\Sigma_{a b}(\lambda) \delta r_{b} e^{\lambda t},
$$

where the synaptic kernel (Eq. 2) in frequency domain is given by

$$
\Sigma_{a b}(\lambda)=\frac{e^{-\lambda D_{a b}}}{1+\lambda \tau_{d}} .
$$

From Eq. 5, we obtained the perturbation of total input to population $a$

$$
\delta I_{a}(t)=J_{a 1} \delta s_{a 1}(t)+J_{a 2} \delta s_{a 2}(t)-J_{a 3} \delta s_{a 3}(t) .
$$

Finally, the new output rate of the network in response to the input rate perturbation is given by

$$
\delta r_{a} e^{\lambda t}=R_{a}(\lambda) \delta I_{a}(t)
$$

Similar to the synapse, neuron population also acts like a frequency filter which can written as, for the rate model,

$$
R_{a}^{\mathrm{RM}}(\lambda)=\frac{1}{\tau_{a}(1+\lambda \omega)}
$$

and, for LIF neurons, $R_{a}^{\mathrm{FP}}$ can be either calculated numerically using the threshold integration method [22] or by hypergeometric functions $[3,5]$.

Combining Eqs. 5, 7 and 8, we obtained a system of three linear equations in $\delta r_{a}, a=1,2,3$, which has non-trivial solutions when the determinant of following matrix is zero:

$$
0=\left|\begin{array}{ccc}
-1 & A_{12}(\lambda) & -A_{13}(\lambda) \\
A_{21}(\lambda) & -1 & -A_{23}(\lambda) \\
A_{31}(\lambda) & A_{32}(\lambda) & -\left(1+A_{33}(\lambda)\right)
\end{array}\right|
$$

where $A_{a b}(\lambda)=J_{a b} \Sigma_{a b}(\lambda) R_{a}(\lambda)$. Rearranging Eq. (10), we obtained

$$
\begin{aligned}
0 & =\left(1-A_{12} A_{21}\right)(1+\underbrace{A_{33}}_{J_{33} \text { coupling }}) \\
& +\underbrace{A_{32} A_{23}+A_{31} A_{12} A_{23}}_{J_{23} \text { coupling }} \quad \text { (Fast inhibition) } \\
& +\underbrace{A_{31} A_{13}+A_{32} A_{21} A_{13}}_{J_{13} \text { coupling }} \quad \text { (Slow inhibition) }
\end{aligned}
$$

Eq. 11 describes network motifs in the $E_{1} E_{2} I_{3}$ model that can induce network oscillations. The two first lines of Eq. 11 consist of motifs that receive fast inhibition with short delay $d$ via two different pathways. The local inhibitory coupling $J_{33}$ is responsible for generating oscillatory activity via the monosynaptic $A_{3} A_{3}$ loop, whereas the inhibitory coupling $J_{23}$ is responsible for generating oscillatory activity via disynaptic $A_{32} A_{23}$ and trisynaptic $A_{31} A_{12} A_{23}$ loops. The third line of Eq. 11 consists of network motifs that receive slow inhibition. In this case, the inhibitory coupling $J_{13}$ is responsible for generating oscillatory activity via disynaptic $A_{31} A_{13}$ and trisynaptic $A_{32} A_{21} A_{13}$ loops with long delay $D$. In the following we systematically investigate how the slow and fast inhibitory pathways interact to shape the oscillatory dynamics of the three population network.

\section{B. Transition to oscillatory states}

To obtain analytical estimates of a Hopf bifurcation, which marks the transition from a steady state to an oscillatory state, we substituted $\lambda=i \omega$ into Eq. 11 to find bifurcation points as a function of $D$ and $J_{33}$ (or $D$ and $J_{23}$ ). A similar calculation was performed in [5]. We denoted the amplitude and the phase of the population response function $R_{a}(i \omega)$ as $H_{a}$ and $\phi_{a}$, respectively, i.e. $R_{a}(i \omega)=H_{a}(\omega) \exp \left(-i \phi_{a}(\omega)\right)$. The amplitude and the phase of synaptic kernel $\Sigma_{a b}(i \omega)$ were denoted as $H_{s}$ and $\phi_{s}$, respectively, i.e. $\Sigma_{a b}=H_{s}(\omega) \exp \left(-i \phi_{s}(\omega)-\right.$ $\left.i D_{a b} \omega\right)$ where $D_{a b} \omega$ is the phase shift due to a transmission delay. To simplify notations, we let $\Phi_{a}=\phi_{a}+\phi_{s}$ be the sum of phase shifts due to population response of $a$ and synaptic dynamics (without a delay), and $\Phi_{a b}=\Phi_{a}+\Phi_{b}$ etc.

The real part of Eq. 11 is

$$
\begin{aligned}
0 & =1-c_{r} \cos \left(\Phi_{12}+2 D \omega\right) \\
& +c_{i}\left[\cos \left(\Phi_{3}+d \omega\right)-c_{r} \cos \left(\Phi_{123}+2 D \omega+d \omega\right)\right] \\
& +c_{p_{d}} \cos \left(\Phi_{23}+2 d \omega\right)+c_{p_{t}} \cos \left(\Phi_{123}+2 D \omega+d \omega\right) \\
& +c_{t} \cos \left(\Phi_{123}+2 D \omega+d \omega\right)+c_{d} \cos \left(\Phi_{13}+2 D \omega\right)
\end{aligned}
$$

and its imaginary part is

$$
\begin{aligned}
0 & =-c_{r} \sin \left(\Phi_{12}+2 D \omega\right) \\
& +c_{i}\left[\sin \left(\Phi_{3}+d \omega\right)-c_{r} \sin \left(\Phi_{123}+2 D \omega+d \omega\right)\right] \\
& +c_{p_{d}} \sin \left(\Phi_{23}+2 d \omega\right)+c_{p_{t}} \sin \left(\Phi_{123}+2 D \omega+d \omega\right) \\
& +c_{t} \sin \left(\Phi_{123}+2 D \omega+d \omega\right)+c_{d} \sin \left(\Phi_{13}+2 D \omega\right)
\end{aligned}
$$

where $c_{r}=J_{12} J_{21} H_{1} H_{2} H_{s}^{2}$ is the bidirectional coupling between $E_{1}$ and $E_{2}, c_{i}=J_{33} H_{3} H_{s}$ is the $I_{3}-I_{3}$ coupling, $c_{p_{d}}=J_{32} J_{23} H_{2} H_{3} H_{s}^{2}$ and $c_{p_{t}}=J_{31} J_{12} J_{23} H_{1} H_{2} H_{3} H_{s}^{3}$ are the disynaptic and trisynaptic $E_{2}-I_{3}$ couplings, respectively, and $c_{d}=J_{13} J_{31} H_{1} H_{3} H_{s}^{2}$ and $c_{t}=J_{32} J_{21} J_{13} H_{1} H_{2} H_{3} H_{s}^{3}$ and the disynaptic and trisynaptic $E_{1}-I_{3}$ couplings, respectively.

In the following calculations, $J_{33}$ and $D$ are the two bifurcation parameters, and we sought to express them as functions of $\omega$ (See Appendix A for the derivation of other critical couplings). First, to write $J_{33}$ as a function of $\omega$, we removed $D$ from Eqs. 12 and 13 by moving three (two) terms in Eq. 12 (Eq. 13) that did not include $D$ to the other side of the equation, squaring both sides of each equation, then adding two equations to obtain a quadratic equation of $c_{i}$,

$$
0=A c_{i}^{2}+2 B c_{i}+C \text {. }
$$


Then,

$$
c_{i}^{ \pm}=\frac{1}{A}\left(-B \pm \sqrt{B^{2}-A C}\right)
$$

or

$$
J_{I_{3} I_{3}}^{ \pm}=c_{i}^{ \pm} /\left(H_{3} H_{s}\right)
$$

where

$$
\begin{gathered}
A=c_{r}^{2}-1 \\
B=-c_{r}\left(c_{p_{t}}+c_{t}\right)+\left(c_{r}^{2}-1\right) \cos \left(\Phi_{3}+d \omega\right) \\
-\left(c_{r} c_{d}+c_{p_{d}}\right) \cos \left(\Phi_{2}+d \omega\right) \\
C=2\left[\left(c_{p_{t}}+c_{t}\right)\left(-c_{r} \cos \left(\Phi_{3}+d \omega\right)+c_{d} \cos \left(\Phi_{2}+d \omega\right)\right)\right. \\
\left.-c_{p_{d}} \cos \left(\Phi_{23}+2 d \omega\right)-c_{r} c_{d} \cos \left(\Phi_{3}-\Phi_{2}\right)\right] \\
+c_{r}^{2}-c_{p_{d}}^{2}+c_{p_{t}}^{2}+c_{d}^{2}+c_{t}^{2}+2 c_{p_{t}} c_{t}-1 .
\end{gathered}
$$

Next, to write $D$ as a function of $\omega$, we invoked trigonometric identities in Eqs. 12 and 13 to derive a system of equations to explicitly solve for $\cos 2 D \omega$ and $\sin 2 D \omega$.

$$
\left[\begin{array}{ll}
M_{11} & M_{12} \\
M_{21} & M_{22}
\end{array}\right]\left[\begin{array}{l}
\cos 2 D \omega \\
\sin 2 D \omega
\end{array}\right]=\left[\begin{array}{l}
P \\
Q
\end{array}\right]
$$

where

$$
\begin{gathered}
M_{11}=-M_{22}=-c_{r} \cos \Phi_{12}-c_{r} c_{i} \cos \left(\Phi_{123}+d \omega\right) \\
+c_{p_{t}} \cos \left(\Phi_{123}+d \omega\right)+c_{d} \cos \Phi_{13}+c_{t} \cos \left(\Phi_{123}+d \omega\right) \\
M_{12}=M_{21}=c_{r} \sin \Phi_{12}+c_{r} c_{i} \sin \left(\Phi_{123}+d \omega\right) \\
-c_{p_{t}} \sin \left(\Phi_{123}+d \omega\right)-c_{d} \sin \Phi_{13}-c_{t} \sin \left(\Phi_{123}+d \omega\right) \\
P=-1-c_{i} \cos \left(\Phi_{3}+d \omega\right)-c_{p_{d}} \cos \left(\Phi_{23}+2 d \omega\right) \\
Q=c_{i} \sin \left(\Phi_{3}+d \omega\right)+c_{p_{d}} \sin \left(\Phi_{23}+2 d \omega\right) .
\end{gathered}
$$

Substituting $c_{i}^{ \pm}$calculated above, we obtained an expression for the lateral delay for Hopf bifurcation

$$
D^{ \pm}=\frac{1}{2 \omega} \operatorname{atan}\left(\frac{-M_{21}^{ \pm} P^{ \pm}+M_{11}^{ \pm} Q^{ \pm}}{M_{22}^{ \pm} P^{ \pm}-M_{12}^{ \pm} Q^{ \pm}}\right) \text {. }
$$

In the following sections, we describe the Hopf bifurcation lines $\left(D^{ \pm}, J_{33}^{ \pm}\right)$that satisfy both Eqs. 13 and 12 by varying $\omega$ and compare the analytical results with numerical solutions of delay differential equations for the rate model and simulations of networks of leaky integrate-and-fire neurons for the spiking network model.

\section{DYNAMICAL STATES OF $E_{1} E_{2} I_{3}$ NETWORK}

In the following, we refer to the (non-oscillatory) steadystate as $S$ and three oscillatory states as $O_{1}, O_{2}$ and $O_{3}$. As described in Eq. 11, three types of inhibitory couplings $J_{13}, J_{23}$ and $J_{33}$ are responsible for generating the oscillatory states $\mathrm{O}_{1}, \mathrm{O}_{2}$ and $\mathrm{O}_{3}$, respectively.
A.i

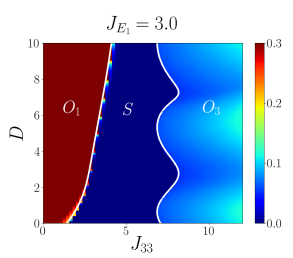

B.i

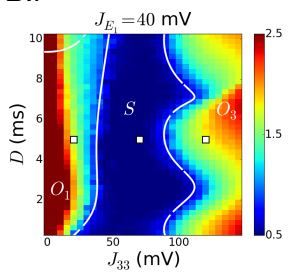

A.ii

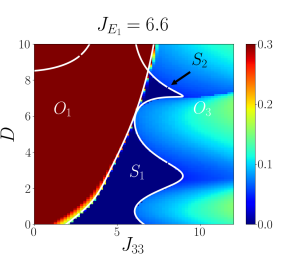

B.ii

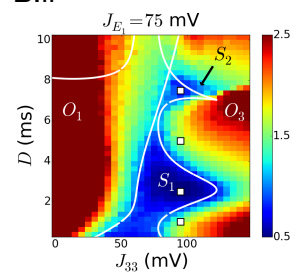

A.iii

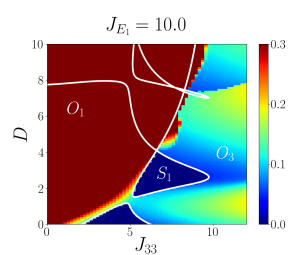

B.iii

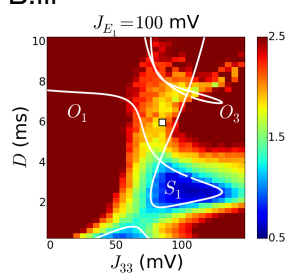

FIG. 2. Bifurcation diagrams of (A) rate model and (B) spiking network model as a function of $I_{3}-I_{3}$ coupling $J_{33}$ and lateral delay $D$. The strength of synaptic projection, $J_{E_{1}}\left(=J_{21}, J_{31}\right)$, from $E_{1}$ to $E_{2} I_{3}$ is weak (A.i, B.i), intermediate (A.ii, B.ii) and strong (A.iii, B.iii). For the rate model, $J_{12}=0.5, J_{13}, J_{23}, J_{32}=2$, $d=2.5, \tau_{d}=0$. For the spiking network model, the total (individual) synaptic weights $J_{12}=30(0.03), J_{13}=80(0.32), J_{23}, J_{32}=$ $50(0.2,0.05) \mathrm{mV}$. Note that the weights of individual synapses from neuron $i$ in population $a$ to neuron $j$ in population $b$ is given by $J_{a b} /\left(p N_{b}\right)$ (Eq. 3), so that $I_{3}$ to $I_{3}$ synaptic weights are between 0 and $0.6 \mathrm{mV}$. Local delay $d=2.5 \mathrm{~ms}$, and synaptic decay time $\tau_{d}=1 \mathrm{~ms}$. Color bars show the coefficient of variations of the network models (See text for details). White lines show analytical estimates of a Hopf bifurcation (Eqs. 14 and 15).

\section{A. Network activity states}

To characterize different dynamical states of the three population network, we systematically varied the $I_{3}-I_{3}$ coupling $\left(J_{33}\right)$ and the delay in lateral connections $(D)$ (Fig 2). For each parameter pair $\left(J_{33}, D\right)$, we simulated the network for 1.2 seconds and measured the standard deviation of the population rates to estimate whether the network exhibited oscillations. The external input to each population was adjusted to maintain constant population rates across different network setups $(5 \mathrm{~Hz}$ for excitatory and $10 \mathrm{~Hz}$ for inhibitory spiking neurons) while other network parameters remained fixed. For both network models, the standard deviation of each population rate was normalized by its means and averaged over three populations to obtain the coefficient of variation of the population activity. When the network was oscillating, the standard deviation of the network activity was higher as the network activity waxed and waned. This is, however, only an indirect measure and may not directly imply oscillations, therefore, we also examined the spectrum of the population activity (Fig. 3).

For a fixed value of $D$, the network exhibited three distinct states, $\mathrm{O}_{1}, \mathrm{~S}$ and $\mathrm{O}_{3}$, as we varied $J_{33}$. (Hopf bifurcation estimates are shown as white lines in Fig. 2). The estimates of the network activity states closely matched with the states obtained from a corresponding simulations of a network of spiking neurons (Figs. 2A, B). For low values of $J_{33}, O_{1}$ was 
observed which is characterized by slow frequency oscillations $(\approx 25 \mathrm{~Hz})$ mediated by the $E_{1}-I_{3}$ loop with lateral delay $D=5 \mathrm{~ms}$ (see white squares in Fig. 2B.i and the corresponding spiking activity in Fig. 3A, top). For moderate values of $J_{33}$, oscillations vanished and a non-oscillatory state emerged $(S)$ in which neurons fired asynchronously and the population firing rate randomly fluctuated around the steady-state (akin to the asynchronous irregular state of [3]). Finally for very strong inhibitory coupling (high $J_{33}$ ), the non-oscillatory state was transformed into another oscillatory state $O_{3}$, which was characterized by high frequency oscillations $(\approx 100 \mathrm{~Hz})$ mediated by the local $I_{3}-I_{3}$ loop with local delay $d=2.5 \mathrm{~ms}$.

As the lateral delays $D$ was increased the regions of $O_{1}$ monotonically creased and the state $S$ was observed at higher values of $J_{33}$. The lateral delays $D$ had a more dramatic effect on the emergence of the state $O_{3}$. The value of $J_{33}$ at which the oscillatory state $O_{3}$ was observed varied in a periodic manner as $D$ was increased (see the interface of $O_{1}$ and $S$ and that of $O_{3}$ and $S$ in Figs. 2A.i and B.i). While, the oscillation frequency in $O_{1}$ state decreased monotonically with $D$, the oscillation frequency in $O_{3}$ state was independent of $D$ (Fig 3A, bottom; black lines: analysis, red squares: simulations).

To better understand why the critical $J_{33}$ weights varied in a periodic manner as a function of lateral delays $D$, we next examined the oscillation dynamics at the interface of the nonoscillatory state $S$ and the oscillatory state $O_{3}$

\section{B. Modulation of critical $I_{3}-I_{3}$ coupling due to lateral delay}

To gain intuition on why the local coupling $J_{33}$ may depend on lateral delays $D$, we reduced the subnetwork $E_{2} I_{3}$ to an inhibitory population $I_{3}$. This effectively assumes that the subnetwork $E_{2} I_{3}$ operates in an inhibition dominated regime. By solving Eqs. 12 and 13 for the critical $I_{3}-I_{3}$ coupling in terms of $D$ we obtained

$$
J_{33}=\frac{\left(1-2 c_{d}(\omega) \cos \left(\Phi_{13}+2 D \omega+\pi\right)+c_{d}(\omega)^{2}\right)^{1 / 2}}{H_{3}(\omega) H_{s}(\omega)} .
$$

From Eq. 16, the reduced network model suggests that the critical $J_{33}$ modulates periodically as a function of $D$, and the period of modulation $T=\pi / \omega^{*}=1 /\left(2 f^{*}\right)$ is determined by the frequency of network oscillations, $\omega^{*}=2 \pi f^{*}$. (Here we assumed that the oscillation frequency at the transition to $\mathrm{O}_{3}$ state depends weakly on $D$; in other words, $\omega(D) \equiv \omega^{*}$ on the boundary of $O_{3}$ as shown in Fig. 3A (bottom).) Moreover, the maximum of $J_{33}$ is attained at

$$
(2 n+1) \pi=\Phi_{13}\left(\omega^{*}\right)+2 D \omega^{*}+\pi,
$$

and the minimum of $J_{33}$ at

$$
2 n \pi=\Phi_{13}\left(\omega^{*}\right)+2 D \omega^{*}+\pi
$$

for $n=0, \pm 1, \ldots$, where $\Phi_{13}\left(\omega^{*}\right)+2 D \omega^{*}$ is the total phase shift induced by the bidirectional lateral connections between
A

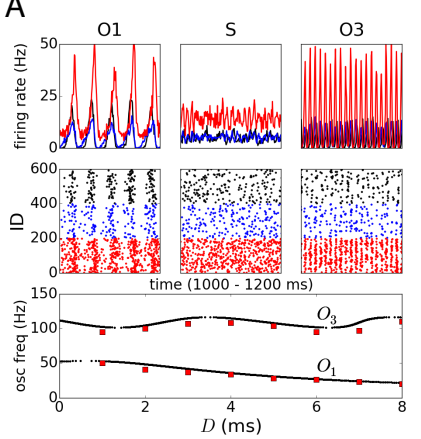

$\mathrm{B}$

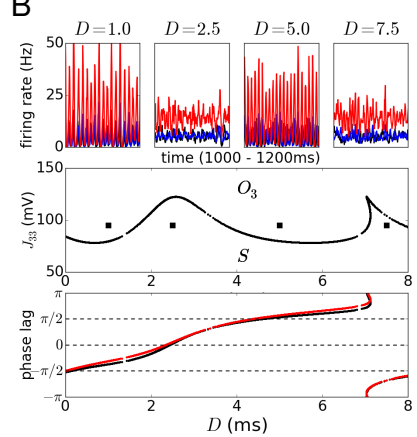

C
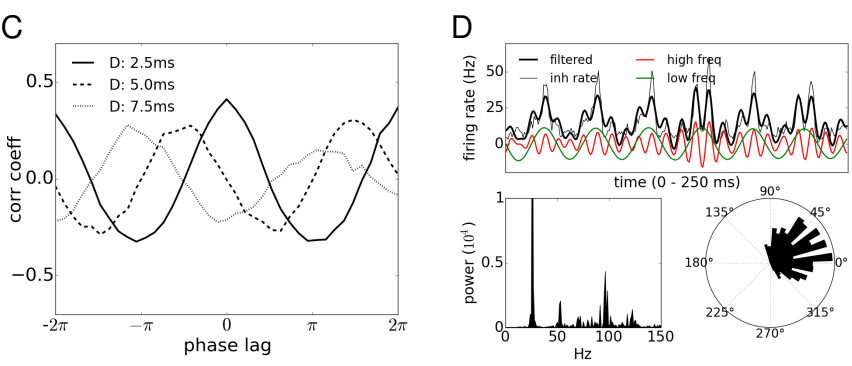

FIG. 3. (A) (top) Instantaneous firing rate of $E_{1}$ (black), $E_{2}$ (blue) and $I_{3}$ (red) populations in $S, O_{1}$ and $O_{3}$ states; (middle) Spike raster of the corresponding spiking activity. (bottom) Oscillation frequency of $O_{1}$ and $O_{3}$ states as a function of lateral delay; black: analysis, red: simulations (B) (top) Alternation between $S$ and $O_{3}$ states due to the lateral delay, corresponding to white squares in Fig. 2B.ii. (middle) Analytical estimates of critical $J_{I_{3} I_{3}}$ (black line) and network states corresponding to the top row (black squares). (bottom) Analytical estimate of the phase lag $\Delta \phi_{31}$ of $I_{3}$ with respect to $E_{1}$ in the $O_{3}$ state; black: full network (Eq. (B1)), red: simplified $E_{1} I_{3}$ network (Eq. (19)). (C) Cross-correlation of mean firing rate of $E_{1}$ and $I_{3}$, calculated using simulation results from part (B). (D) Cross-frequency coupling appears when $O_{1}$ and $O_{3}$ merge; (top) mean firing rate of $I_{3}$ (gray), its high (red) and low (green) frequency components, and the sum of high and low frequencies (black); (left bottom) power spectrum of $I_{3}$ mean firing rate; (right bottom) Correlation between fast oscillation amplitude (black bar) and slow oscillation phase (angle).

$E_{1}$ and $I_{3}$, and $\pi$ appears due to the inhibitory coupling. In other words, Eq. 17 (Eq. 18) suggests that the critical $J_{33}$ reaches its maximum (minimum) when the network oscillation relayed through the lateral connections is anti-phase (inphase) to the oscillations generated in $I_{3}$.

Conceptually, this phenomenon can be understood as follows. If oscillatory activity relayed through lateral connections is in-phase with oscillatory activity generated by the local $E_{2}-I_{3}$ loop, weak coupling is sufficient to induce networkwide oscillations. On the other hand, if the relayed network activity is anti-phasic to locally generated activity, stronger coupling is required to overcome the suppression of the local activity by the relayed activity. Because changing the lateral delay shifts the phase of relayed activity continuously, the critical coupling strength modulates qusi-periodically with a period determined by the oscillation frequency. In Fig. 2B.i, for instance, the period of $\mathrm{O}_{3}$-boundary ( $T \approx 5 \mathrm{~ms}$ ) is determined 
by the oscillation frequency of $O_{3}$ state $(f \approx 100 \mathrm{~Hz}$, Fig. 3A, bottom): $T \approx 1 /(2 f)$ as predicted by Eq. 16 .

We also calculated the relative phase $\Delta \phi_{31}^{\max }\left(\Delta \phi_{31}^{\min }\right)$ of $I_{3}$ population rate with respect to the $E_{1}$ population rate at the maximum (minimum) of $J_{33}$. For the reduced network model, the phase lag is given by

$$
\Delta \phi_{31}=-\pi+\Phi_{1}+D \omega,
$$

and at the max and min of $J_{33}$,

$$
\begin{aligned}
\Delta \phi_{31}^{\max } & =(n-1) \pi, \\
\Delta \phi_{31}^{\min } & =\left(n-\frac{1}{2}\right) \pi, \quad n=0, \pm 1, \pm 2, \ldots
\end{aligned}
$$

where we used Eqs. 17 and 18 to evaluate $D \omega^{*}$ and assumed that the phase shifts due to population response functions are equal, i.e. $\Phi_{1}=\Phi_{3}$. We then verified numerically that the phase lag in the full $E_{1} E_{2} I_{3}$ network can be approximated by that of the reduced network (Fig. 3B, bottom; black: full network, red: reduced network). See Appendix B for the derivation of phase lags in the full and reduced network models.

\section{Cross-frequency coupling}

The network architecture investigated here has been suggested to generate cross-frequency oscillations in which power in a frequency band is modulated by the phase of another oscillation [13]. In our model, multi-frequency oscillations appeared as we increased the strength of long range excitation $J_{E_{1}}$.

At an intermediate step towards the emergence of multifrequency oscillations, we examined how the strength of connections from the excitatory population $E_{1}\left(J_{E_{1}}\right)$ affected the landscape of the network states. First, we found that increase in $J_{E_{1}}$ increased the region of the state $O_{1}$. Second, $J_{E_{1}}$ also increased the modulation in the boundary of state $\mathrm{O}_{3}$. Together, these changes meant that the region of non-oscillatory state was not observed for some values of $D$, such that the non-oscillatory state appeared in isolated regions, $S_{1}$ and $S_{2}$ in Figs. 2A.ii and B.ii. There was a wide range of inhibitory coupling $J_{33}$ (e.g. $80-120 \mathrm{mV}$ in Fig. 2B.ii), over which the network can switch between non-oscillatory and oscillatory states by varying the lateral delay (white squares in Fig. 2B.ii and the corresponding spike activity shown in Fig. 3B, top and middle).

As the strength of synaptic input from $E_{1}$ was further increased, two Hopf bifurcation lines defining the $O_{1}$ and $O_{3}$ states merged and created a small region in the space spanned by $J_{33}$ and $D$ in which the network exhibits non-oscillatory activity $S_{1}$ (Fig 2A.iii and B.iii). Outside of $S_{1}$, where $O_{1}$ and $\mathrm{O}_{3}$ merged (e.g. white square in Fig. 2B.iii), the slow oscillatory activity induced by the lateral $E_{1}-I_{3}$ loops and the fast oscillatory activity induced by the local $I_{3}-I_{3}$ loop coexisted. This was evident in the power spectrum of inhibitory population firing rate, which showed two peaks at low $(25 \mathrm{~Hz})$ and high $(100 \mathrm{~Hz})$ frequencies (Fig. 3D, left bottom). Moreover, when the population rates were band-passed filtered at
A.i

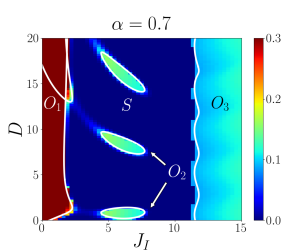

B.i

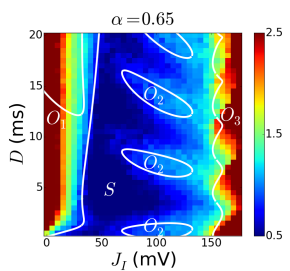

ii

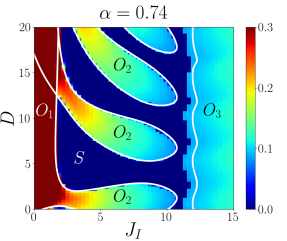

ii

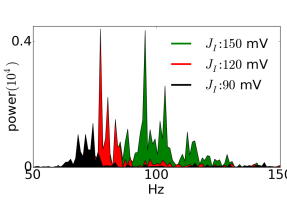

iii

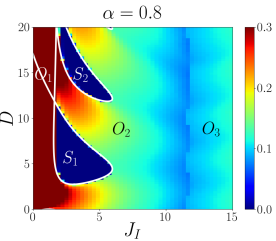

iii

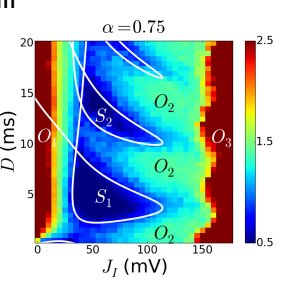

FIG. 4. Composition of three oscillatory states. $\alpha$ denotes the relative strength of $E_{2}-I_{3}$ loop: $J_{23}=\alpha J_{I}, J_{33}=J_{I}$ (A) Rate model; $J_{12}=0.5, J_{13}=2, J_{32}=4, J_{21}=J_{31}=1, d=2.5$, $\tau_{d}=0$. (B) Spiking network model; B.ii, Power spectrum of population $I_{3}$ 's mean firing rates for $D=7 \mathrm{~ms}$ in B.i.; Total (individual) synaptic weights, $J_{12}=30(0.03), J_{13}=60(0.24), J_{32}=$ $120(0.12), J_{21}=J_{31}=30(0.06) \mathrm{mV}, d=2.5 \mathrm{~ms}, \tau_{d}=1$ ms. Color bars show the coefficient of variations of network models. White lines show analytical estimates of a Hopf bifurcation (Eqs. A2 and 15).

high and slow frequencies then summed up, the filtered population rates closely followed the actual inhibitory firing rates (Fig. 3D, top). Interestingly, in our model the amplitude of fast oscillation was modulated according to the phase of slow oscillation (Fig 3D, right bottom). This is akin to the modulation of gamma band osculations power by the phase of theta oscillations [23] in the hippocampus.

Thus, we show that when a partially overlapping neuron population participates in generation of both fast oscillation and slow oscillation (i.e. $I_{3}$ is part of the fast $I_{3}-I_{3}$ loop and the slow $E_{1}-I_{3}$ loop), cross-frequency coupling emerges in which slow oscillation generated via the lateral loop $\left(E_{1}-I_{3}\right)$ with long delay $D$ modulates the amplitude of fast oscillation $\left(I_{3}-I_{3}\right)$ by providing periodic input.

\section{Emergence of an oscillatory state driven by $E_{2}-I_{3}$ coupling}

Thus far, we considered oscillatory activity generated by the $I_{3}-I_{3}$ loop. In this section, we describe how including the oscillatory activity due to the $E_{2}-I_{3}$ loop further enriches the dynamical landscape of the three population network. To control the interaction of the $E_{2}-I_{3}$ and $I_{3}-I_{3}$ loops, we introduce a parameter $\alpha$ that determines the relative strength of the $E_{2}-I_{3}$ coupling with respect to $I_{3}-I_{3}$ coupling: $J_{23}=\alpha J_{I}$, $J_{33}=J_{I}$.

With weak coupling between $E_{2}-I_{3}$ (i.e. small $\alpha$ ), Hopf bifurcation structure was identical to the one obtained by varying $J_{33}$ alone, as shown in Figs. 2A.i and 2B.i. In other words, the $O_{3}$ state, driven by the $I_{3}-I_{3}$ loop, was the only oscillatory 
state that can be generated by the $E_{2} I_{3}$ subnetwork. When $\alpha$ was increased, a new oscillatory state $\mathrm{O}_{2}$, driven by the $E_{2}$ $I_{3}$ loop, emerged in the small isolated regions in the space spanned by $J_{1}$ and $D$ (Figs. 4A.i and B.i). The $O_{2}$ state emerged in a parameter space which led to the non-oscillatory state $(S)$ for low values of $\alpha$. That is, for high $\alpha$ values, long-range interactions with the population $E_{1}$ destabilized the non-oscillatory state of the $E_{2} I_{3}$ subnetwork to create oscillations.

To better understand why non-oscillatory and different oscillatory states appear as a function of $J_{I}$, we fixed the lateral delay (e.g. $D=9$ in Fig. 4A.i) and examined the changes in network state as $J_{I}$ increases. For small $J_{I}$, local inhibition was too weak to withstand the oscillatory instability driven by the lateral $E_{1}-I_{3}$ loop, so the network entered the $O_{1}$-state. The network shifted to the non-oscillatory state $S$ when the local inhibition $J_{I}$ was increased. When $J_{I}$ became sufficiently strong to generate oscillatory activity through the $E_{2}-I_{3}$ loop, the network entered $O_{2}$-state. When $J_{I}$ was further increased, the increased $I_{3}-I_{3}$ coupling suppressed the oscillatory activity induced by the $E_{2}-I_{3}$ loop, which brought the network back to the non-oscillatory state. For strong $J_{I}$, the $I_{3}-I_{3}$ coupling becomes the dominant network motif producing oscillatory activity, hence the network entered the $\mathrm{O}_{3}$ state.

The $\mathrm{O}_{2}$-state, however, appeared only within a restricted range of lateral delays (e.g. $7<D<10$ in Fig. 4A.i) and vanished gradually outside of this range. As discussed in Section IV B, the $\mathrm{O}_{2}$ state appears when the oscillatory activity relayed through the lateral connections is in-phase with the ongoing oscillations in the $E_{2} I_{3}$ subnetwork. On the other hand, the $\mathrm{O}_{2}$ state can no longer exist when the relayed network activity no longer enhances the local activity. Such resonance and cancellation effects, occurring repeatedly, gave rise to isolated $\mathrm{O}_{2}$-states at multiple sites. The periodic appearance of stationary and oscillatory states is a robust phenomenon in delayed feedback system, which has been investigated in the context of controlling pathological brain rhythms $[18,20]$.

When the $E_{2}-I_{3}$ coupling became stronger ( $\alpha$ was increased), the $\mathrm{O}_{2}$ state expanded across the non-oscillatory state $S$ and merged with the $O_{1}$ state, creating a complex configuration of multiple network states as shown in Fig. 4A.ii. The $O_{1}$ and $O_{3}$ states, previously separated by $S$, were now bridged by an elongated $O_{2}$ region. When $\alpha$ was further increased, all three oscillatory states, $O_{1}, O_{2}$ and $O_{3}$, appeared contiguously, and the non-oscillatory states formed isolated regions surrounded by the $O_{1}$ and $O_{2}$ states (Figs. 4A.iii and B.iii). Such dynamical landscape is similar to the previously discussed bifurcation structure that produced cross-frequency oscillations at a parameter region where multiple Hopf bifurcations meet (see Figs. 2 A.iii and B.iii).

In networks of spiking neurons, $\mathrm{O}_{2}$-states emerged from the non-oscillatory state at multiple sites, as predicted by the analysis. However, unlike the dynamical landscape of the firing rate model (Fig. 4A.i), network simulations showed that $\mathrm{O}_{2}$ states did not exist in isolation. The network activity remained oscillatory in-between the $\mathrm{O}_{2}$ and $\mathrm{O}_{3}$ states (Fig. 4B.i), and the oscillation frequency increased gradually as the network transitioned from $\mathrm{O}_{2}, \mathrm{~S}$, to $\mathrm{O}_{3}$ (Fig 4B.ii). When $\alpha$ became large as shown in Fig. 4B.iii, the $\mathrm{O}_{2}$ state expanded horizontally across the non-oscillatory state, connected the $O_{1}$ and $O_{3}$ states, and created isolated non-oscillatory states surrounded by the oscillatory states, $O_{1}$ and $O_{2}$, similarly to the firing rate model (Fig. 4A.iii).

\section{v. CONCLUSION}

Population of neurons embedded in a larger network rarely acts alone but interacts in concert with neighboring neurons to produce network activity. In this study we extended the standard two-population model consisting of excitatory and inhibitory neurons, and demonstrated in a minimal threepopulation model that when an excitatory-inhibitory network is coupled to an additional excitatory population, the lateral connections between them can create a rich bifurcation structure, composed of isolated stationary states, multiple oscillatory states and cross-frequency coupling. Such dynamical landscape allows the network to fix its operating point in the non-oscillatory stationary state and easily tap into various oscillatory states generating slow frequency oscillations (via lateral $E_{1}-I_{3}$ loop), fast frequency oscillations (via local $E_{2}-I_{3}$ and $I_{3}-I_{3}$ loops), or nested (cross-frequency) oscillations (by positioning itself in a region where slow and fast oscillations merge). We also found that two types of local oscillations $\left(\mathrm{O}_{2}\right.$ and $\left.\mathrm{O}_{3}\right)$ can be interwoven intricately with the stationary states, which has not been observed in isolated excitatoryinhibitory networks.

Previously, the coupling of gamma $(30-80 \mathrm{~Hz})$ and theta (4-12 Hz) frequency oscillations in the hippocampus was investigated using a network model composed of one excitatory and two types of inhibitory neuron models, where one of the inhibitory neuron type (O-LM interneuron) was responsible for generating the gamma rhythm [24]. Our results, as suggested in [13], demonstrate a different mechanistic model for cross-frequency coupling where there is only one type of inhibitory neuron but two modes of oscillations can be generated via long lateral and short local delays.

Various effects of time delay on neural dynamics have been studied extensively in neural field equation that models spatial interactions. It has been shown that the delay can induce oscillations for local excitation-lateral inhibition interaction [25], give rise to rich bifurcation structure in simple scalar model [26], and stabilize stochastic bump attractors [27].

Our results demonstrate that a lumped firing rate model and randomly connected spiking models can also develop rich dynamic repertoire without any spatial interactions.

To cleary expose the rich dynamics of the three population network we have used rather wide range of delays $(0-20 \mathrm{~ms})$. Long delays beyond $10 \mathrm{~ms}$ are usually not observed in biological neuronal networks. All the key dynamical states $\left(O_{1}\right.$, $S, S_{1}, O_{2}, O_{3}$ can be observed for lateral delays up to $5 \mathrm{~ms}$ which are known to exist in the brain. For instance, the thalamocortical loop has $3.4 \mathrm{~ms}$ delay in mice [28]) and the interhemispheric connections can show delays of $3-9 \mathrm{~ms}$ in different species [29]. In primates inter-hemispheric delays are 
about $4 \mathrm{~ms}$ [30]. Our results show that slow conductation delays in thalamocortical loops and inter-hemispheric connections can play a very important role in reshaping the local network dynamics and introducing new dynamics through the long range interactions. Our network simulations and analysis was restricted to a special case in which all delays were fixed. It would be of interest to study the effects of distributed delays in the future, as in [31-33], to reflect biologically realistic connectivity and go beyond the three population motifs.

\section{ACKNOWLEDGMENTS}

This work was supported by the BrainLinks-BrainTools Cluster of Excellence funded by the German Research Foundation (DFG EXC 1086), the German Federal Ministry of Education and Research (FKZ 01GQ0830), and the INTERREG IV Rhin superieur program and European Funds for Regional Development through the project TIGER A31.

\section{Appendix A: Calculation of critical couplings}

We substitute $\lambda=i \omega$ and calculate the rate response function $R_{a}(i \omega)$ and the synaptic kernel $\Sigma_{a b}(i \omega)$. For the rate model,

$$
\begin{gathered}
R_{a}^{\mathrm{RM}}(i \omega)=1 /\left(1+i \tau_{a} \omega\right)=C_{a} \exp \left(-i \phi_{a}\right) \\
C_{a}=\frac{1}{\sqrt{1+\tau_{a}^{2} \omega^{2}}}, \quad \phi_{a}=\operatorname{atan}\left(\tau_{a} \omega\right),
\end{gathered}
$$

with $0 \leq \operatorname{atan}\left(\tau_{a} \omega\right)<\pi / 2$, and for the spiking network,

$$
\begin{gathered}
R_{a}^{\mathrm{SN}}(i \omega)=\tau_{m a} R_{a}^{\mathrm{THIN}}(i \omega)=C_{a} \exp \left(-i \phi_{a}\right) \\
C_{a}=\tau_{m a}\left|R_{a}^{\mathrm{THIN}}\right|, \quad \phi_{a}=-\arg \left(R_{a}^{\mathrm{THIN}}\right)
\end{gathered}
$$

where $R_{a}^{\text {THIN }}$ is the population response function obtained numerically from the threshold integration method [22]. The synaptic kernel

$$
\begin{gathered}
\Sigma_{a b}(i \omega)=\exp \left(-i \omega D_{a b}\right) /\left(1+i \omega \tau_{d}\right)=C_{s} \exp \left(-i \phi_{s}-i \omega D_{a b}\right) \\
C_{s}=\frac{1}{\sqrt{1+\omega^{2} \tau_{d}^{2}}}, \quad \phi_{s}=\operatorname{atan}\left(\tau_{d} \omega\right) .
\end{gathered}
$$

with $0 \leq \operatorname{atan}\left(\tau_{d} \omega\right)<\pi / 2$.

Substituting $R_{a}(i \omega)$ and $\Sigma_{a b}(i \omega)$ to Eq. (11) yields

$$
\begin{aligned}
& 0=1-c_{r} \exp \left(-i \Phi_{12}-i 2 D \omega\right) \\
& +c_{i}\left[\exp \left(-i \Phi_{3}-i d \omega\right)-c_{r} \exp \left(-i \Phi_{123}-i 2 D \omega-i d \omega\right)\right] \\
& +c_{p_{0}}\left[c_{p_{1}} \exp \left(-i \Phi_{23}-i 2 d \omega\right)\right. \\
& \left.+c_{p_{2}} \exp \left(-i \Phi_{123}-i 2 D \omega-i d \omega\right)\right] \\
& +c_{t} \exp \left(-i \Phi_{123}-i 2 D \omega-i d \omega\right)+c_{d} \exp \left(-i \Phi_{13}-i 2 D \omega\right)
\end{aligned}
$$

where we decompose $c_{p_{d}}$ and $c_{p_{t}}$ in Eqs. (12) and (13) in terms of $c_{p_{0}}=J_{23} A_{2} A_{s}, c_{p_{1}}=J_{32} A_{3} A_{s}$ and $c_{p_{2}}=$ $J_{31} J_{12} A_{1} A_{3} A_{s}^{2}$ in order to explicitly solve for $J_{23}$ in the following calculations.
To study the bifurcaiton structure when $J_{23}$ are varied, we manipulated Eqs. (12) and (13), as discussed in Section III B, to solve for the inhibitory coupling strength $J_{23}=$ $c_{p_{0}} /\left(A_{2} A_{s}\right)$.

$$
\begin{array}{r}
A c_{p_{0}}^{2}+2 B c_{p_{0}}+C=0 \\
c_{p_{0}}=\frac{1}{A}\left(-B \pm \sqrt{B^{2}-A C}\right)
\end{array}
$$

where

$$
\begin{gathered}
A=c_{p_{2}}^{2}-c_{p_{1}}^{2} \\
B=-c_{r} c_{p_{2}} \cos \left(\Phi_{3}+d \omega\right)+\left(c_{d} c_{p_{2}}-c_{i} c_{p_{1}}\right) \cos \left(\Phi_{2}+d \omega\right) \\
-c_{p_{1}} \cos \left(\Phi_{23}+2 d \omega\right)-c_{r} c_{i} c_{p_{2}}+c_{t} c_{p_{2}} \\
C=c_{r}^{2}+\left(c_{r} c_{i}\right)^{2}+c_{d}^{2}+c_{t}^{2}-c_{i}^{2}-1 \\
+2\left[\left(c_{r}^{2} c_{i}-c_{i}-c_{r} c_{t}\right) \cos \left(\Phi_{3}+d \omega\right)-c_{r} c_{d} \cos \left(\Phi_{3}-\Phi_{2}\right)\right. \\
\left.+\left(c_{t}-c_{r} c_{i}\right) c_{d} \cos \left(\Phi_{2}+d \omega\right)-c_{r} c_{i} c_{t}\right]
\end{gathered}
$$

On the other hand, to study the bifurcation structure when both $J_{23}$ and $J_{33}$ are varied, we similarly manipulate Eqs. (12) and (13) to solve for the inhibitory coupling $J_{I}$ where $J_{23}=$ $\alpha J_{I}$ and $J_{33}=J_{I}$.

$$
\begin{array}{r}
A J_{I}^{2}+2 B J_{I}+C=0 \\
J_{I}=\frac{1}{A}\left(-B \pm \sqrt{B^{2}-A C}\right)
\end{array}
$$

where

$$
\begin{aligned}
& A=\left(c_{r} \bar{c}_{i}-c_{p_{2}} \bar{c}_{p_{0}}\right)^{2}-\bar{c}_{i}^{2}-\left(c_{p_{1}} \bar{c}_{p_{0}}\right)^{2}-2 \bar{c}_{i} c_{p_{1}} \bar{c}_{p_{0}} \cos \left(\Phi_{2}+d \omega\right) \\
& B=\left(c_{r}^{2} \bar{c}_{i}-c_{r} c_{p_{2}} \bar{c}_{p_{0}}-\bar{c}_{i}\right) \cos \left(\Phi_{3}+d \omega\right) \\
& +\left(c_{p_{2}} \bar{c}_{p_{0}} c_{d}-c_{r} \bar{c}_{i} c_{d}\right) \cos \left(\Phi_{2}+d \omega\right) \\
& -c_{p_{1}} \bar{c}_{p_{0}} \cos \left(\Phi_{23}+2 d \omega\right)-c_{r} \bar{c}_{i} c_{t}+c_{p_{2}} \bar{c}_{p_{0}} c_{t} \\
& C=c_{r}^{2}+c_{d}^{2}+c_{t}^{2}-1+2\left[-c_{r} c_{d} \cos \left(\Phi_{3}-\Phi_{2}\right)\right. \\
& \left.-c_{r} c_{t} \cos \left(\Phi_{3}+d \omega\right)+c_{d} c_{t} \cos \left(\Phi_{2}+d \omega\right)\right] \\
& \text { and } \bar{c}_{p_{0}}=\alpha c_{p_{0}} / J_{23} \text { and } \bar{c}_{i}=c_{i} / J_{33} .
\end{aligned}
$$

\section{Appendix B: Phase lag}

From the second and third lines of the coefficient matrix, Eq. (10), we can derive

$$
\delta r_{3}=\frac{A_{31}+A_{32} A_{21}}{1+A_{33}+A_{23} A_{32}} \delta r_{1},
$$

which implies that the relative phase difference

$$
\Delta \phi_{31}=\arg \left[\left(A_{31}+A_{32} A_{21}\right) /\left(1+A_{33}+A_{23} A_{32}\right)\right]
$$

of $I_{3}$ with respect to $E_{1}$ is determined by the later couplings, $A_{31}$ and $A_{32} A_{21}$, that connect $E_{1}$ to $E_{3}$, and the local couplings, $A_{33}$ and $A_{23} A_{32}$. In Fig 3B, we numerically evaluate the above $\Delta \phi_{31}$. 
On the other hand, for the reduced two-population network considered in Section IV B, the first line of Eq. (10),

$$
\delta r_{3}=-\frac{1}{A_{13}} \delta r_{1},
$$

gives a simple expression for the phase difference

$$
\Delta \phi_{31}=-\pi+\Phi_{1}+D \omega
$$

[1] C. van Vreeswijk and H. Sompolinsky, Science 274, 1724 (1996).

[2] C. van Vreeswijk and H. Sompolinsky, Neural computation 10, 1321 (1998).

[3] N. Brunel, Journal of Computational Neuroscience 8, 183.

[4] N. Brunel and X.-J. Wang, Journal of neurophysiology 90, 415 (2003).

[5] E. Ledoux and N. Brunel, Frontiers in computational neuroscience 5, 25 (2011).

[6] S. Ostojic, Nature neuroscience 17, 594 (2014).

[7] S. Denève and C. K. Machens, Nature Neuroscience 19, 375 (2016).

[8] S. Sadeh, S. Cardanobile, and S. Rotter, SpringerPlus 3, 148 (2014).

[9] B. K. Murphy and K. D. Miller, Neuron 61, 635 (2009).

[10] A. Kumar, S. Rotter, and A. Aertsen, Journal of neuroscience 28, 5268 (2008).

[11] P. Fries, Annual review of neuroscience 32, 209 (2009).

[12] G. Hahn, A. F. Bujan, Y. Frégnac, A. Aertsen, and A. Kumar, PLoS computational biology 10, e1003811 (2014).

[13] A. Hyafil, A. L. Giraud, L. Fontolan, and B. Gutkin, Trends in Neurosciences 38, 725 (2015).

[14] A. Palmigiano, T. Geisel, F. Wolf, and D. Battaglia, Nature neuroscience 20, 1014 (2017).

[15] A. Roxin, N. Brunel, and D. Hansel, Physical review letters 94, 238103 (2005).

[16] A. Roxin and E. Montbrió, Physica D: Nonlinear Phenomena 240, 323 (2011).

[17] J. Kremkow, A. Aertsen, and A. Kumar, Journal of neuroscience 30, 15760 (2010).

[18] M. Rosenblum and A. Pikovsky, Physical Review E - Statistical, Nonlinear, and Soft Matter Physics 70, 1 (2004).

[19] O. V. Popovych, C. Hauptmann, and P. A. Tass, Physical Re- view Letters 94, 164102 (2005).

[20] I. Vlachos, T. Deniz, A. Aertsen, and A. Kumar, PLoS Computational Biology 12, 1 (2016).

[21] M.-O. Gewaltig and M. Diesmann, Scholarpedia 2, 1430 (2007).

[22] M. J. E. Richardson, Physical Review E 76, 021919 (2007).

[23] L. L. Colgin, T. Denninger, M. Fyhn, T. Hafting, T. Bonnevie, O. Jensen, M.-B. Moser, and E. I. Moser, Nature 462, 353 (2009).

[24] A. B. L. Tort, H. G. Rotstein, T. Dugladze, T. Gloveli, and N. J. Kopell, Proceedings of the National Academy of Sciences of the United States of America 104, 13490 (2007).

[25] A. Hutt, Physics Letters A 372, 541 (2008).

[26] R. Veltz, SIAM Journal on Applied Dynamical Systems 12, 1566 (2013).

[27] Z. P. Kilpatrick, Physica D: Nonlinear Phenomena 295, 30 (2015).

[28] Z. V. Guo, H. K. Inagaki, K. Daie, S. Druckmann, C. R. Gerfen, and K. Svoboda, Nature 545, 181 (2017).

[29] K. A. Phillips, C. D. Stimpson, J. B. Smaers, M. A. Raghanti, B. Jacobs, A. Popratiloff, P. R. Hof, and C. C. Sherwood, Proc. R. Soc. B 282, 20151535 (2015).

[30] R. Caminiti, F. Carducci, C. Piervincenzi, A. Battaglia-Mayer, G. Confalone, F. Visco-Comandini, P. Pantano, and G. M. Innocenti, Journal of Neuroscience 33, 14501 (2013).

[31] C. G. Assisi, V. K. Jirsa, and J. A. S. Kelso, Physical review letters 94, 018106 (2005).

[32] F. M. Atay and A. Hutt, SIAM Journal on Applied Dynamical Systems 5, 670 (2006).

[33] S. Petkoski, A. Spiegler, T. Proix, P. Aram, J.-J. Temprado, and V. K. Jirsa, Physical Review E 94, 012209 (2016). 